First-line-Therapie mit Bevacizumab bei nichtkleinzelligem Lungenkarzinom

\title{
Auch ältere Patienten und Patienten mit Begleiterkrankungen können profitieren
}

Das zeigen die Ergebnisse einer Interimsanalyse der prospektiven Phase-IV-Studie ARIES, die bei der Jahrestagung der American Society of Clinical Oncology (ASCO) 2010 in Chicago/USA vorgestellt wurden (Wozniak AJ et al., ASCO 2010: \#7618 and poster presentation).

In der ARIES-Studie werden die Sicherheit und Verträglichkeit von Bevacizumab (Avastin ${ }^{\circledR}$ ) unter Alltagsbedingungen untersucht. Dabei werden auch Patientengruppen erfasst, die in randomisierten Studien oft unterrepräsentiert sind, wie beispielsweise ältere Patienten, Patienten mit schlechtem Allgemeinzustand (Eastern Cooperative Oncology Group Performance Status [ECOG PS] >2) oder Patienten mit ZNS-Metastasen.

Für die aktuelle Interimsanalyse wurden die Daten von 1.967 Patienten mit fortgeschrittenem NSCLC, die mindestens eine Dosis Bevacizumab erhalten hatten, berücksichtigt. 32,9\% der Patienten waren $\geq 70$ Jahre alt, $18,8 \%$ der Patienten sogar $\geq 75$ Jahre alt. Diese älteren Patienten hatten häufiger einen schlechten Allgemeinzustand (ECOG PS >2) als jüngere Patienten (<70 Jahre) und oft kardiovaskuläre Begleiterkrankungen, die medikamentös behandelt wurden. Insgesamt hatten 7,6\% der Patienten ZNS-Metastasen, $7,1 \%$ der Patienten Hämoptysen und 9,3\% der Patienten einen schlechten Allgemeinzustand (ECOG PS >2). 49,1\% der Patienten hatten weiterhin einen zentral lokalisierten Tumor und bei $14,6 \%$ der Patienten war eine Kavitation im Tumor nachweisbar.

\section{PFS in allen Patientengruppen vergleichbar}

Nach einer medianen Beobachtungszeit von 11,3 Monaten betrug das mediane progressionsfreie Überleben (PFS) aller Patienten 6,6 Monate und das mediane Gesamtüberleben (OS) 13,3 Monate. Das mediane PFS war in allen Patientengruppen vergleichbar. Das mediane Gesamtüberleben war bei älteren Patienten (>70 Jahren) mit 12,2 Monaten etwas niedriger als in der Gruppe der Patienten $<70$ Jahre.

Die Häufigkeit unerwünschter Ereignisse unterschied sich nicht von der in randomisierten Studien. Bei Patienten mit ZNS-Metastasen wurden keine ZNS-Blutungen $>$ Grad 3 beobachtet. Blutungen $>$ Grad 3 - pulmonale/gastrointestinale Blutungen, Epistaxis und andere Blutungen - traten bei 3,6\% der Patienten auf; sie wurden bei älteren und jüngeren Patienten gleich häufig beobachtet. Drei der 217 Patienten mit Kavitationen hatten eine Lungenblutung $>$ Grad 3. Gastrointestinale Perforationen traten bei 20 Patienten (1\%) auf; sie waren bei älteren Patienten und Patienten in schlechtem Allgemeinzustand nicht häufiger. Arterielle Thromboembolien (ATE) waren in allen Subgruppen gleich häufig, mit Ausnahme der Gruppe der älteren Patienten $(3,2 \%)$ und bei Patienten mit schlechtem Allgemeinzustand (3,3\%); bei diesen Patienten traten ATE häufiger auf als bei jüngeren Patienten und bei Patienten mit gutem Allgemeinzustand (1,2\% bzw. 1,7\%).

Die Ergebnisse der Interimsanalyse bestätigen die Verträglichkeit und auch die Wirksamkeit von Bevacizumab bei Patienten mit fortgeschrittenem NSCLC unter Alltagsbedingungen. Viele Patienten, die zuvor aufgrund von Risikoabwägungen von einer Therapie mit Bevacizumab ausgeschlossen wurden, können diesen Daten zufolge ebenfalls von dem Angiogenesehemmer profitieren. Weitere ASCO-Daten zur NSCLC-Therapie mit Bevacizumab stellt Martin Reck, Großhansdorf, unter www.nsclc.avastin.de vor.

Quelle: Informationen der Roche Pharma AG

\section{Chronische Lymphatische Leukämie}

\section{Alemtuzumab plus Fludarabin}

Hochrisikopatienten mit rezidivierter bzw. refraktärer chronischer lymphatischer Leukämie (CLL) profitieren besonders von der Kombination Alemtuzumab plus Fludarabin (FluCAM). Das zeigen die Ergebnisse der Zwischenauswertung der CAM314-Studie nach median 21 Monaten Follow-up (Engert A et al., EHA 2010: Poster 0768).

In der offen randomisierten CAM314-Studie wurde untersucht, ob die Hinzunahme von Alemtuzumab zu einem Fludarabin-haltigen Schema (FluCAM) im Vergleich zur Fludara-
bin-Monotherapie bei vorbehandelten $\mathrm{Pa}$ tienten mit rezidierter bzw. refraktärer CLL zusätzliche Vorteile bietet. Im primären Endpunkt, dem progressionsfreien Überleben, zeigte sich in der aktuellen Auswertung ein signifikanter Vorteil zugunsten der Kombination (median 24,1 Monate vs. 15,4 Monate; $p=0,001)$. Dabei profitierten Patienten $>65$ Jahre und Patienten mit hohem Risiko (Stadium Rai III-IV) besonders. Bei den Hochrisikopatienten zeigte sich sogar ein Vorteil im Gesamtüberleben $(p<0,005)$.

Quelle: Informationen der Genzyme GmbH

\author{
Misteltherapie: Broschüre \\ für Fachkreise \\ Die Broschüre "Iscador ${ }^{\circledR}$ in der Tu- \\ mortherapie - Empfehlungen für \\ die Behandlung" der Weleda AG \\ informiert umfassend zur erwei- \\ terten Tumortherapie mit dem an- \\ throposophischen Mistelpräparat. \\ Im Mittelpunkt stehen dabei die \\ Therapieempfehlungen für jede \\ Tumorlokalisation und die prak- \\ tische Durchführung der Therapie. Die \\ Broschüre kann von Fachkreisen kosten- \\ frei bestellt werden: E-Mail: kundenser- \\ vice@weleda.de, Art.-Nr. 50661000.
}

